\title{
Rapid Colonoscopy Preparation Using Bolus Lukewarm Saline Combined with Sequential Posture Changes: A Randomized Controlled Trial
}

\author{
Vijaypal Arya $\cdot$ Kalpana A. Gupta $\cdot$ \\ Ashok Valluri $\cdot$ Swarn V. Arya $\cdot$ Martin L. Lesser
}

Received: 13 October 2012/Accepted: 7 February 2013/Published online: 2 March 2013

(c) The Author(s) 2013. This article is published with open access at Springerlink.com

\begin{abstract}
Objective In this randomized clinical trial, we have compared the Shudh ${ }^{\mathrm{TM}}$ colon cleanse (SCC) with HalfLytely ${ }^{\circledR}$ colon prep (HCP) to evaluate the efficacy, bowel preparation time (BPT), adverse events, electrolyte abnormalities and patient acceptability.

Methods Patients were randomized to receive either SCC $(n=65)$ or HCP $(n=68)$. All colonoscopies were performed by a single, blinded endoscopist. Colon prep was evaluated on a 5 point grading scale. Statistical non-inferiority was predefined as a difference of $<15 \%$ in the lower limit of the
\end{abstract}

Presented at: ACG 2009—Presidential Poster Award Recipient, San Diego, CA. ACG 2010, San Antonio, TX.

Electronic supplementary material The online version of this article (doi:10.1007/s10620-013-2598-9) contains supplementary material, which is available to authorized users.

\section{Arya $(\bowtie)$}

75-54 Metropolitan Ave, Midldle Village, NY 11379, USA

e-mail: varyamd@yahoo.com; av336@cornell.edu

V. Arya

Weill Medical College of Cornell University, Newyork,

NY, USA

\section{Arya}

Division of Gastroenterology/Hepatobiliary Diseases,

Wyckoff Heights Medical Center, Brooklyn, NY, USA

V. Arya

Brooklyn Hospital, Brooklyn, NY, USA

V. Arya

North Shore University Hospital, Manhasset, NY, USA

K. A. Gupta

Methodist Hospital, Brooklyn, NY, USA

e-mail: vjkal2000@yahoo.com
95.5\% confidence interval for the treatment difference. Data that were collected include bowel prep score, BPT, adverse events, electrolyte abnormalities and patient acceptability.

Results Bowel preparation efficacy was rated as "successful" for 59/65 (90.7\%) in SCC versus 66/68 (97.1\%) in HCP. This gave a success difference of $-6.4 \%$ with a 1-sided $95 \%$ lower confidence limit (LCI) for the difference $=-13.3 \%$ (non-inferiority $p=0.25$ ). This difference fell within the predefined limit for non-inferiority. The average BPT for SCC was $1.9 \mathrm{~h}$ versus 10.9 in HCP $(p<0.001)$. No serious adverse events were reported in either group. None of the patients in either group had any clinically significant electrolyte imbalance. Patient ratings for palatability and willingness to repeat were significantly better for SCC $(p<0.05)$.

Conclusion SCC was found to not be inferior to PEG with regards to the quality of bowel preparation. It is worth

\author{
A. Valluri \\ Wyckoff Heights Medical Center, Brooklyn, NY, USA \\ e-mail: valluri.ashok@gmail.com \\ S. V. Arya \\ Cornell University, Ithaca, NY, USA \\ e-mail:sva8@cornell.edu \\ M. L. Lesser \\ Biostatistics Unit, Feinstein Institute for Medical Research, \\ Manhasset, NY, USA \\ M. L. Lesser \\ Department of Molecular Medicine, Hofstra North Shore-LIJ \\ School of Medicine, New Hyde Park, NY, USA \\ M. L. Lesser \\ Department of Population Health, Hofstra North Shore-LIJ \\ School of Medicine, New Hyde Park, NY, USA
}


highlighting that a major advantage of SCC is shorter BPT. (Clinical trial registration number: NCT01547130).

Keywords Colon preparation - Saline $\cdot$ Posture

\section{Introduction}

Colon cancer is the third most common cancer in the United States and is the second leading cause of cancer deaths [1]. Importantly, colon neoplasm is one of the most preventable cancers, and regular colonoscopy screening could prevent most deaths due to this cancer by early detection and removal of both cancer and precancerous polyps [2]. Despite this evidence, screening rates are disturbingly low. Nationally, only about $35 \%$ of Americans aged 50 years and older have ever undergone colonoscopy [3]. From a patient perspective, several factors influence this low rate of screening, including low household income, lack of health insurance, being of Hispanic or Asian decent, limited access to care, and no physician recommendation to be screened [4].

A quality colonoscopy examination remains as the gold standard for colorectal cancer screening [5, 6]. Although it is a valuable diagnostic tool, many eligible patients are intimidated by the idea of a colonoscopy. For those patients who are able and willing to undergo the colonoscopy procedure, colon cleansing is a major barrier to meticulous mucosal examination from rectum to cecum [7, 8]. Polyethylene glycol (PEG) based preps have been used as the major bowel preparation for colonoscopy in the United States. However, the total volume of intake, prep duration, and palatability continue to be major patient concerns. These factors influence patient acceptability and subsequently the preparation outcome [9, 10]. An ideal colon prep should be safe, effective, fast, palatable and economical. Additionally, it should have minimal dietary restrictions and should not interrupt sleep. To date, no single prep has been consistently superior for all groups of patients [11]. The search for an ideal prep continues.

The use of complementary and alternative medicine in the United States is on the rise [12, 13] The beneficial effects of alternative therapies on cardiovascular, neuroendocrine, and other systems have been reported [14-19]. Thus we endeavored to apply the principles of mindful meditation in order to ascertain its effects in colonoscopy preparation. Bolus drinking of lukewarm saline with sequential posture changes is an alternative process that has been used for internal cleansing. We standardized this

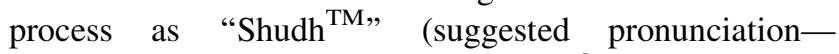
"should") and compared with NuLytely ${ }^{\circledR}$ [20]. In addition to mindfulness, there are two active components in SCC: A-Bolus (8 oz/240 $\mathrm{mL}$ in 1-2 min) drinking of lukewarm normal saline and B-sequential posture changes (SPC) of mild intensity (metabolic equivalent value of three [21]) with deep breathing.

Although it appears to be simplistic, the innovative aspect of SCC focuses on a bolus intake of saline solution in quick succession (every 4-5 $\mathrm{min}$ ) rather than intermittent sipping (Fig. 1). It is theorized that a bolus intake versus intermittent sipping should lead to a larger amount of gastric emptying (first order kinetics-with a time to $50 \%$ emptying of $8-18 \mathrm{~min}$ for isotonic, non-nutritive liquids [22-24]).When two meals are taken within a few minutes, the gastric emptying of the second meal is faster than that of the first $[22,25]$. The saline load is known to stimulate the gastro-colic reflex [26]. Simultaneously, the resultant high flow rate of isotonic solution in the intestine should allow minimal time for ionic exchange, leading to less absorption.

We propose a model that explains the plausible mechanism of action (Fig. 2). Inputs from sensory sources are modified by cognition and effect, and then integrated in the central nervous system (CNS), autonomic nervous system (ANS) and enteric nervous system (ENS) with an outcome of effects on gastro-intestinal (GI) motility, secretion and blood flow [27]. In SCC, thinking to get clean (mindfulness), deep breathing (pulmonary stretch reflex) [28], bolus drinking [23-25] of isotonic lukewarm saline [29, 30], and SPC (mild intensity exercise [31], gravity and posture [3234]) activate the CNS, parasympathetic component of ANS, and ENS. This leads to gastric receptive relaxation, gastro-colic reflex and increased GI motility. A detailed description of these mechanisms is beyond the scope of this article. It is however well known that psychological factors (stress and relaxation) do influence the gut physiology in irritable bowel syndrome [35]. It is reasonable to conclude that in SCC, concentration and relaxation (mindfulness) will have a positive role in bowel cleansing. Saline purgatives (magnesium sulfate and sodium chloride ions) are known to increase the motor activity of the small and large bowel, which could be secondary to the release of cholecystokinin-pancreozymin (CCK-PZ) [36].

Our pilot study found that SCC was as effective as NuLytely ${ }^{\circledR}$ in colon preparation. Furthermore, it found that preparation could be achieved in 101 min with SCC [20]. The present study was conducted in order to compare SCC to HCP with regard to efficacy, bowel prep time, adverse events, electrolyte abnormalities and patient acceptability.

\section{Methods}

This was a randomized, single-center, single-blinded (to the endoscopists) and active-control study. Patients undergoing 


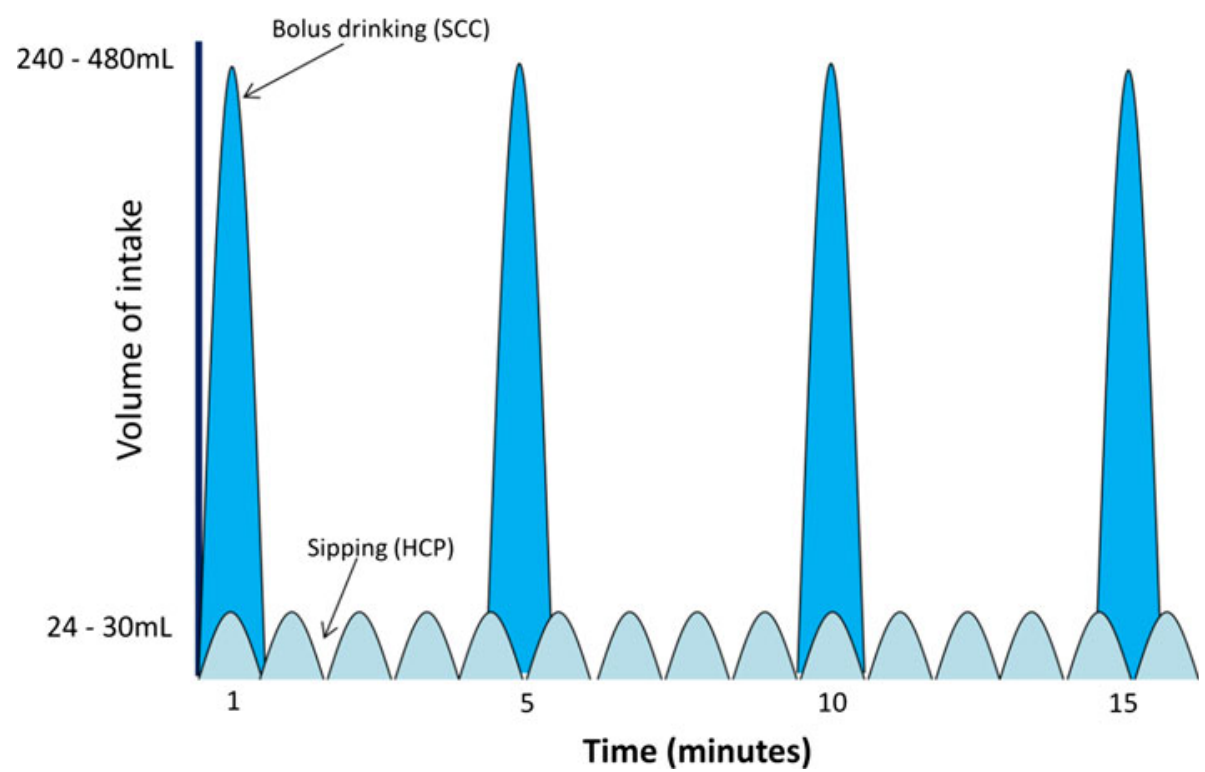

Fig. 1 Method of drinking: Bolus versus sipping Bolus drinking of normal saline (SCC group) in quick succession (every 4-5 min) allows intake of 1-2 L where as sipping of PEG solution (HCP group) allows intake of only $360-450 \mathrm{~mL}$ in $15 \mathrm{~min}$. Bolus intake every 4-5 min should lead to a larger amount of gastric emptying (applying first order kinetics for a time to $50 \%$ emptying of 8-18 min for an isocaloric, non-nutrient liquid), thus stimulating a potent gastro-colic reflex. Simultaneously, the resultant high flow rate of isotonic solution in the intestine should allow minimal time for ionic exchange, leading to less absorption

\section{INPUT \\ INTEGRATION}

1) Thinking to get clean
2)Deep breathing -
pulmonary stretch reflex
3) Bolus drinking of
lukewarm saline in
quick succession
4) Yoga poses

1) Thinking to get clean 2)Deep breathing -

3) Bolus drinking of Barm saline in 4) Yoga poses

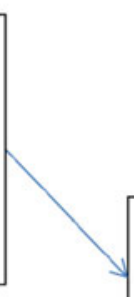

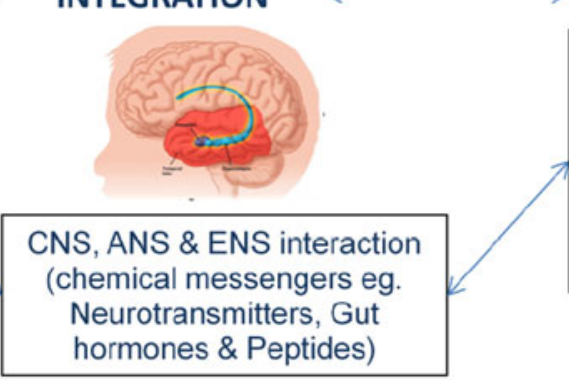

\section{EFFECT}

Parasympathetic stimulation:

1) Relaxation of $\mathrm{GI}$ sphincters

2) Faster gastric emptying

3) Gastro-colic reflux

4) Increased intestinal motility

5) CCK-PZ release

Brain, lungs and GI tract - areas of inputs, Brain, ANS and ENS - Areas of integration and GI tract Areas of effects

Fig. 2 Hypothetical mechanism of action (Shudh model)

elective colonoscopy between May 2008 and December 2010 were recruited. The protocol was approved by the Institutional Review Board (Wyckoff Heights Medical Center, an affiliate of Weill Cornell Medical School, NY).

\section{Patients}

All patients included in the study provided written informed consent. Patients 21-70 years of age who were referred for colonoscopy with good general physical status (American Society of Anesthesiologists (ASA) class 1 or 2) and who expressed a willingness to perform SCC were included in the study. Patients were excluded if they had a history of chronic heart, liver, or kidney disease; hypertension, diabetes mellitus, arthritis (spine, shoulder, hip and knee joints) severe constipation, or concurrent severe diarrhea. Additional exclusion criteria included ileus, suspected intestinal obstruction, bowel perforation, previous gastrointestinal tract surgery, gastro-paresis, toxic colitis, ulcerative colitis, pregnancy, and lactation.

\section{Study Design}

Patients were randomized to receive either SCC or HCP using a computer generated random number list. The numbers were assigned consecutively by a research nurse when the appointment for colonoscopy was given. The same nurse, who was trained in SCC, explained the bowel preparation procedures to the patients and was available for any further questions. However, the patients were instructed to maintain the confidentiality of his/her bowel preparation assignment with all other office staff and the endoscopist. 
Weight and vital signs were recorded before and after the bowel preparation. Blood samples were collected at first visit and again after completion of the bowel preparation (prior to colonoscopy) to analyze serum levels of sodium, potassium, chloride, carbon-dioxide, blood urea nitrogen, creatinine and glucose (BMP).

After preparation but before colonoscopy, patients from both groups completed a symptom questionnaire where they rated (1-5 Liekert scale) solution palatability and willingness to repeat the preps. Patients in the SCC group were additionally asked to rate the ease of SPC. Adverse events were rated by both groups in the same questionnaire.

\section{Study Materials and Medications}

SCC (Shudh ${ }^{\mathrm{TM}}$, Vikalp Inc., NY) package contained a 32-oz plastic pitcher and 9-gm salt sachets (Iodine-free table saltUSP grade sodium chloride) for preparation of $0.9 \%$ saline (1 sachet in 32-oz lukewarm water $\left(37.2-38.8^{\circ} \mathrm{C}\right.$ or 99-102 $\left.{ }^{\circ} \mathrm{F}\right)$ ), an instructional leaflet, and a DVD providing instructions of the bowel preparation process. Patients were instructed to fill the pitcher with 16-oz of hot water and $16-\mathrm{oz}$ of room temperature water to reach the lukewarm temperature. Patients could add a twist of lemon if the solution was unpalatable to them. The HCP (HalfLytely ${ }^{\circledR}$ ) (Braintree Laboratories, MA) is a Food and Drug Administration approved bowel preparation before colonoscopy. It comes as a white powder for reconstitution containing $210 \mathrm{~g}$ of PEG-3350, $2.86 \mathrm{~g}$ of sodium bicarbonate, $5.6 \mathrm{~g}$ of sodium chloride, $0.74 \mathrm{~g}$ of potassium chloride and 1 gram of flavoring ingredient (optional) to be reconstituted in $2 \mathrm{~L}$ water and two bisacodyl delayed release tablets. Flavor Packs are available in Cherry, Lemon-Lime and Orange.

\section{Preparation Instructions}

\section{Shudh ${ }^{T M}$ Colon Cleanse (SCC)}

The instructions for the SCC were provided in the DVD and explained by the research nurse onsite at the first visit. The DVD included dietary instructions and information on the relaxation process/meditation, how to prepare the solution, sequential posture changes and 'how to drink' in bolus form (Appendix A). Patients were instructed to view the DVD and practice the postures at least 1 day in advance to become familiar with the process. The instructions were also provided in the form of a leaflet.

One day prior to the colonoscopy, patients receiving the SCC were instructed to eat a light breakfast menu meal (e.g. toast, boiled or scrambled egg, cereal, oatmeal, milk, juice, tea, coffee) up to 12 p.m. After that, they were instructed to stay on clear liquids (water, apple juice, grape juice, Gatorade, clear broth, popsicles, Jell-O, and coffee or tea without milk or non-dairy creamer) until they went to bed. They were instructed to stay nil per os (NPO) after midnight. Patients were suggested to start the SCC around 6 a.m. on the morning of the colonoscopy and to drink their last glass of solution at least $2 \mathrm{~h}$ before the scheduled procedure as per American Society of Anesthesiologists (ASA) and American Society of Gastrointestinal Endoscopy (ASGE) guidelines $[37,38]$. They were instructed to wear comfortable, loosefitting clothing, turn off phones and pagers, select a quiet environment with an available dedicated bathroom, and to relax. Patients were instructed to concentrate on the breath entering and leaving the nostrils, while thinking to get clean (turning the mind away from outside distractions and focusing inwards-mindful bowel cleansing).

Patients in the SCC group took a bolus intake of $8 \mathrm{oz}$. $(240 \mathrm{~mL})$ or $16 \mathrm{oz} .(480 \mathrm{~mL})$ of lukewarm saline water alternating with posture change and followed the steps until clear bowel movement (supplement 1).

\section{HalfLytely ${ }^{\circledR}$ Colon Prep (HCP)}

Patients in the HCP group followed the preparation method according to the manufacturer's standard instructions. Patients were instructed to stay on clear liquids the entire day before the colonoscopy. Two tablets of bisacodyl delayed-release tablets with water were taken at around 1:00 p.m. Patients were instructed to start drinking the solution after a bowel movement or around 7 p.m. if no bowel activity occurred. They were instructed to sip all of the solution at a rate of $8 \mathrm{oz}$. every $10 \mathrm{~min}$.

\section{Outcome Measures}

The primary endpoint was the "success" rate of the preparations, and the secondary end points were BPT, adverse events, electrolyte abnormalities and preparation acceptability. Preparation efficacy was evaluated by a single, blinded endoscopist (V.A.), who performed all of the colonoscopies. The evaluation involved the rating of six anatomical segments of the colon (rectum, sigmoid, descending colon, transverse colon, ascending colon and cecum) on the 5 point Arya Bowel Prep Scale (ABPS) (Table 1). Aggregating the segmental scores resulted in overall scores. Grade A was defined as a total overall score of $19-24$, grade $B$ as a score of $13-18$, grade $C$ as a score of $7-12$, and grade D as a score of 0-6. Grade A or B preparation was considered "successes", while grade C or D was considered "failures." To assess the reliability of ABPS, we trained 4 gastroenterologists and 3 fellows with the description as well as the endoscopic images corresponding to the 5-point scale (0-4). Ten colonoscopy videos were randomly selected which had scores ranging from 12 to 24 . These DVDs were then evaluated and scored 
Table 1 Arya bowel prep scale (ABPS)

\begin{tabular}{ll}
\hline Score & Description \\
\hline 0-Very poor & Solid or semisolid stool in one of the predefined areas, unable to complete the procedure \\
1-Poor & Semi-solid/thick stool adherent to the mucosa, poor visibility after wash/suck, able to complete the procedure \\
2-Suboptimum & Particles of adherent stool, partial visibility after wash/suck \\
3-Optimum & Few particles of stool/puddles of see through stool, complete visibility after wash/suck \\
4-Excellent & No remaining particles of stool, no need to wash/suck, complete visibility \\
\hline
\end{tabular}

The evaluation involved the rating of six anatomical segments of the colon (rectum, sigmoid, descending colon, transverse colon, ascending colon and cecum) on a 5 point scale. Each segment receives a score of $0-4$. The maximum ABPS score could be 24, representing an excellent prep. The minimum ABPS score could be 0, representing unpreped colon, unable to complete the procedure. Aggregating the segmental scores resulted in overall scores. Grade A was defined as a total overall score of 19-24, grade B as a score of 13-18, grade C as a score of 7-12, and grade D as a score of 0-6. Grade A or B preparation is considered "successes," while grade C or D is considered "failures"

by them separately. The validation results for the 4 attending and 3 fellows were as follows: intraclass correlation $=0.73$ (Inter-rater agreement). The same scale was used successfully in a pilot study [20].

We defined BPT as the time from starting the preparation medication until clear bowel movement instead of until leaving home as described by Jonas et al. [39, 40]. For the SCC group, the BPT started from the first drink, while in the HCP group, it started from pill intake.

\section{Statistical Analysis}

The non-inferiority of SCC was compared with HCP, with pre-specified margins. For prep quality, we estimated a success rate of $85 \%$ in the HCP group [41]. The noninferiority margin was set at $-15 \%$. This means SCC will be considered non-inferior if the difference in success rates (SCC minus HCP) is greater than $-15 \%$. The $-15 \%$ margin of non-inferiority is identical to that used in 4 previously published randomized non-inferiority trials comparing various colon preps [42-44]. The study was designed to have $90 \%$ power to establish non-inferiority when the two treatment groups are equivalent (i.e., the success rates are equal) using a one-tailed test at the $5 \%$ significance level. The required sample size was 70 per group. For the primary outcome variable (or primary end-point), one-sided $95 \%$ confidence interval (CI) and one sided $p$ value against the non-inferiority margin were computed. For the secondary end points, superiority of SCC was compared with HCP.

Analysis of efficacy was carried out using the intentionto-treat (ITT) method. For the ITT analysis, all patients were included in the group that they were assigned to, irrespective of whether they completely followed the study instructions or took preparation doses. Since there was no data from those who cancelled colonoscopy, they were subsequently excluded. Secondary end points were analyzed using the Wilcoxon rank sum or Wilcoxon signed rank test as appropriate. The analysis was carried out using SAS 9.1 (SAS institute, Cary, North Carolina).

\section{Results}

Patient Disposition, Demographics, and Baseline Characteristics

Patient disposition is shown in Fig. 3 and baseline demographics are summarized in Table 2. Out of the 445 patients screened, a total of 147 patients were randomized to receive the SCC $(n=74)$ and the HCP $(n=73)$. Subsequently, 9 patients in SCC and 5 patients in HCP cancelled their procedure for unknown reasons and were lost to follow up. Thus, the final analyses included 65 patients in SCC and 68 patients in HCP. Baseline demographics and characteristics were similar for the two groups.

\section{Preparation Efficacy}

The success rate in the SCC group was $90.7 \%(59 / 65)$ and $97.1 \%(66 / 68)$ in the HCP group, for a difference of $-6.4 \%$ (Table 3). The lower bound for the one-tailed $95 \%$ CI was $-13.2 \%$, well above the $-15 \%$ margin of inferiority. The corresponding $p$ value was $p<0.019$. Thus, SCC was judged to be non-inferior to HCP.

The mean total prep scores were similar in both groups (SCC $18.7( \pm 5.3 \mathrm{SD})$ vs. HCP $20.1( \pm 3.1), p=0.23)$. It is well known that quality of bowel prep varies between segments and the total prep score can be misleading. Hence individual segmental analysis was also done which revealed that the segmental scores were not different in both groups except the rectum which was better in HCP $(p<0.03)$ (Table 4).

\section{BPT and Prep Volume}

The BPT for SCC was 1.25-4 h (average $1.9 \mathrm{~h}$ ), while for HCP was 4-18 h (average $10.9 \mathrm{~h}$ ). The patients in SCC drank an average of $2.7 \mathrm{~L}$ (range 1.2-4 L) as compared to $1.9 \mathrm{~L}$ in $\mathrm{HCP}$ (range 1.5-2 L). 
Fig. 3 Patient disposition

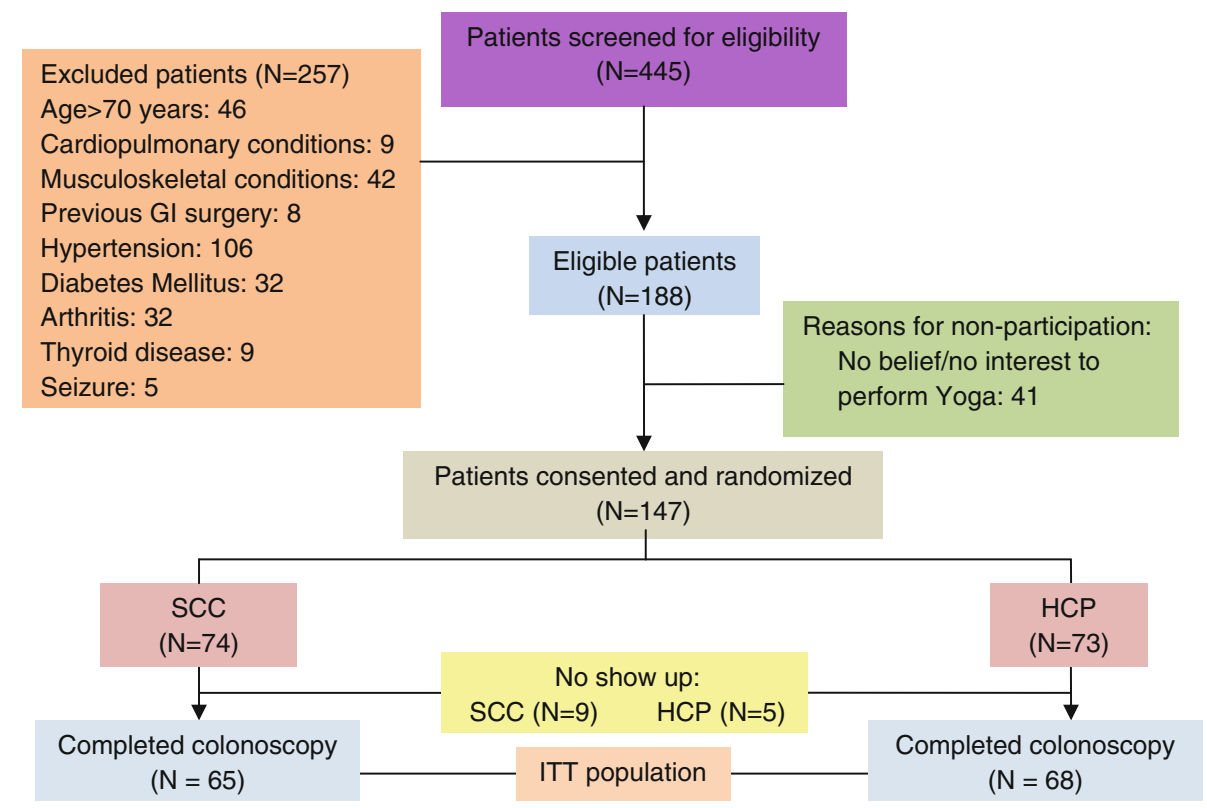

Electrolyte and Weight Changes

Pre- and post-procedural electrolyte analysis is shown in Table 5. A statistically significant decrease in weight was noticed in both groups, although minimal weight gain was also reported in few patients. In the SCC group, the results showed statistically significant elevations from baseline of serum sodium and chloride; and a decrease in bicarbonate and BUN. However, no clinical abnormalities were noticed in any of these patients. Moreover, a slight decrease in BUN indicated the hydrating nature of the SCC. An increase from baseline in sodium and decrease in bicarbonate and BUN of statistical significance was observed in patients in the HCP. However, none of these electrolyte changes resulted in clinical abnormalities.

\section{Adverse Events}

All patients in both groups well tolerated their respective bowel preparations. No patients reported serious adverse events. The common adverse events reported were nausea, vomiting, abdominal cramps, dizziness and headache. In both groups, nausea was the most commonly reported adverse event (25 patients in SCC and 28 patients in HCP). Vomiting was reported significantly more in HCP $(n=12)$ versus the SCC $(n=3 ; p=0.03)$. In SCC, one patient had transient swelling of extremities which relieved same day. Anxiety $(n=1)$, thirst $(n=1)$, burning below the breast bone $(n=1)$ and no bowel movement $(n=1)$ were also experienced in SCC patients. HCP patients experienced chills $(n=2)$, weakness $(n=2)$, hungry feeling $(n=1)$ and no bowel movement $(n=1)$.
Patient Acceptability

Forty-eight (73.9\%) of the 65 responders in the SCC group and $38(55.9 \%)$ of the 68 responders in the HCP group reported that the bowel prep solution was palatable ( $p$ value $0.03)$. Fifty-four $(83.1 \%)$ of the 65 responders in SCC group and $40(58.8 \%)$ of the 68 responders in the HCP group were willing to repeat their respective prep for their future colonoscopies ( $p$ value 0.002) (Table 6). Sixty $(98 \%)$ of the 61 responders in the SCC group perceived that the posture changes were easy to perform.

\section{Discussion}

The results of this randomized study show that for colon cleansing, in healthy patients, SCC was at par with HCP. The major advantage of SCC was rapid cleansing (average $1.9 \mathrm{~h}$ ). Hence SCC can be completed in the morning on the day of the procedure. Following is a typical case scenario:

A 50 year old healthy female was suggested to schedule her colonoscopy. Her medical history was completely unremarkable. After eating a regular breakfast menu meal up to 12 noon, she stayed on clear liquids, completed routine work with no disturbances and had a good night's sleep. She woke up at 6 a.m., performed SCC, finished drinking by 7 a.m. and had a clear bowel movement by 8 a.m.. The colonoscopy procedure was performed at $10 \mathrm{a} . \mathrm{m}$. and she was discharged home at 11 a.m.. The total time 
Table 2 Demographics, indications and baseline characteristics in the ITT population

\begin{tabular}{|c|c|c|c|c|}
\hline & Category & $\operatorname{SCC}(N=65)$ & $\operatorname{HCP}(N=68)$ & Total $(N=133)$ \\
\hline \multicolumn{5}{|l|}{ A. Demographics } \\
\hline \multicolumn{5}{|l|}{ Age (years) } \\
\hline & Mean \pm SD & $43.6 \pm 10.5$ & $44.8 \pm 10$ & $44.2 \pm 10.2$ \\
\hline & 20 to $<40$ & $20(31 \%)$ & $21(31 \%)$ & $41(31 \%)$ \\
\hline & 40 to $<60$ & $43(66 \%)$ & $46(68 \%)$ & $89(67 \%)$ \\
\hline & $\geq 60$ & $2(3 \%)$ & $1(1 \%)$ & $3(2 \%)$ \\
\hline \multicolumn{5}{|l|}{ Sex } \\
\hline & Female & $43(66 \%)$ & $39(57 \%)$ & $82(62 \%)$ \\
\hline & Male & $22(34 \%)$ & $29(43 \%)$ & $51(38 \%)$ \\
\hline \multicolumn{5}{|l|}{ Race } \\
\hline & Caucasian & $55(84.6 \%)$ & $62(91.2 \%)$ & $117(88 \%)$ \\
\hline & African American & $5(7.7 \%)$ & $5(7.4 \%)$ & $10(7.5 \%)$ \\
\hline & Other & $5(7.7 \%)$ & $1(1.4 \%)$ & $6(4.5 \%)$ \\
\hline \multicolumn{5}{|l|}{ B. Indication ${ }^{a}$} \\
\hline & Screening & $24(36.9 \%)$ & $32(47.1 \%)$ & $56(42.1 \%)$ \\
\hline & Mild constipation & $13(20 \%)$ & $12(17.6 \%)$ & $25(18.8 \%)$ \\
\hline & Hematochezia & $14(21.5 \%)$ & $12(17.6 \%)$ & $26(19.5 \%)$ \\
\hline & Abdominal pain & $7(10.8 \%)$ & $7(10.3 \%)$ & $14(10.5 \%)$ \\
\hline & Anemia & $2(3.1 \%)$ & $2(2.9 \%)$ & $4(3 \%)$ \\
\hline & Mild diarrhea & $2(3.1 \%)$ & $2(2.9 \%)$ & $4(3 \%)$ \\
\hline & Other $^{\mathrm{b}}$ & $3(4.6 \%)$ & $1(1.5 \%)$ & $4(3 \%)$ \\
\hline \multicolumn{5}{|l|}{ C. Baseline data } \\
\hline Weight (lbs) & Mean $\pm \mathrm{SD}$ & $155 \pm 29$ & $163 \pm 36$ & $160 \pm 33$ \\
\hline Systolic blood pressure $(\mathrm{mmHg})$ & Mean $\pm \mathrm{SD}$ & $121 \pm 13$ & $125 \pm 17$ & $123 \pm 15$ \\
\hline Diastolic blood Pressure $(\mathrm{mmHg})$ & Mean $\pm \mathrm{SD}$ & $76 \pm 10$ & $80 \pm 10$ & $78 \pm 10$ \\
\hline Sodium (mEq/L) & Mean \pm SD & $140 \pm 2$ & $140 \pm 3$ & $140 \pm 2$ \\
\hline Potassium (mEq/L) & Mean \pm SD & $4.2 \pm 0.4$ & $4.3 \pm 0.3$ & $4.3 \pm 0.3$ \\
\hline Chloride $(\mathrm{mEq} / \mathrm{L})$ & Mean $\pm \mathrm{SD}$ & $104 \pm 2$ & $104 \pm 3$ & $104 \pm 3$ \\
\hline $\mathrm{CO}_{2}(\mathrm{mEq} / \mathrm{L})$ & Mean \pm SD & $23.4 \pm 2.7$ & $23.2 \pm 2.8$ & $23.3 \pm 2.7$ \\
\hline BUN (mEq/L) & Mean \pm SD & $14.3 \pm 3.1$ & $14.6 \pm 4.3$ & $14.4 \pm 3.8$ \\
\hline Creatinine $(\mathrm{mEq} / \mathrm{L})$ & Mean \pm SD & $0.81 \pm 0.16$ & $0.85 \pm 0.18$ & $0.83 \pm 0.17$ \\
\hline Serum glucose (mg/dL) & Mean $\pm \mathrm{SD}$ & $89 \pm 15$ & $92 \pm 15$ & $90 \pm 15$ \\
\hline
\end{tabular}

${ }^{a}$ Multiple indications possible

b Ulcerative colitis ( $n=2$, Yoga), elevated liver function tests $(n=3$, two patients in HalfLytely, one patient in Yoga)

Table 3 Investigator grading of preparation

\begin{tabular}{lccc}
\hline Overall cleaning & SCC N $(\%)$ & HCP N $(\%)$ & Rate difference \\
\hline Success in ITT population (Grade A + B) & $59(90.7 \%)$ & $66(97.1 \%)$ & $-6.4 \%$ \\
& & & $95 \% \mathrm{CI}-13.1 \%-\alpha$ \\
Grade A & $37(56.9 \%)$ & $49(72.1 \%)$ & $-15.2 \%$ \\
Grade B & $22(33.9 \%)$ & $17(25 \%)$ & $+8.9 \%$ \\
Grade C & $2(3.1 \%)$ & $2(2.9 \%)$ & $+0.4 \%$ \\
Grade D & $4(6.2 \%)$ & 0 & $+6.2 \%$
\end{tabular}

Grade A was defined as a total overall score of $19-24$, grade B as 13-18, grade C as 7-12, and grade D as 0-6

she spent on bowel prep was only $2 \mathrm{~h}$. She was highly satisfied with the whole process of colonoscopy prep and the procedure.
The morning only timing of bowel prep provided better quality of sleep, less work disturbance and higher overall satisfaction. The currently available morning only preps are 
Table 4 Segmental and total prep scores

\begin{tabular}{lccc}
\hline & SCC (SD) & HCP (SD) & $p$ value \\
\hline Rectum & $3.40(0.93)$ & $3.74(0.56)$ & 0.03 \\
Sigmoid & $3.40(0.97)$ & $3.59(0.70)$ & 0.37 \\
Left colon & $3.31(0.90)$ & $3.43(0.82)$ & 0.43 \\
Transverse colon & $3.17(1.04)$ & $3.40(0.78)$ & 0.30 \\
Right colon & $2.77(1.22)$ & $2.97(0.93)$ & 0.54 \\
Cecum & $2.63(1.38)$ & $2.96(1.07)$ & 0.24 \\
Total score & $18.69(5.34)$ & $20.10(3.51)$ & 0.23 \\
\hline
\end{tabular}

It is well known that quality of bowel prep varies between segments and the total prep score can be misleading. Hence individual segmental analysis was also done which revealed that the segmental scores were not different in both groups except the rectum which was better in HCP ( $p$ value 0.03 )

good for afternoon colonoscopies because it takes about $8 \mathrm{~h}$ to complete the prep [42]. For day before colonoscopy preps, Jonas et al. [39, 40] reported an average bowel preparation time of $16.5 \mathrm{~h}$. We have demonstrated that healthy patients, who are willing to try alternative method, can achieve bowel prep in $2 \mathrm{~h}$ and can undergo early morning colonoscopy. This is only possible with SCC and not with any other currently available colonoscopy preps.

For all bowel preps, dietary restriction and fasting are two necessary adjuncts. The dietary restriction reduces the amount of solid excreta while the fasting clears the small bowel of all nutrients. Nutrients in the small bowel delay gastric emptying through release of PYY and this is known as ileal break [45]. Theoretically a fasting period of $6-8 \mathrm{~h}$ before starting prep solution will remove ileal break as a hindrance in the success of bowel prep. The presence of nutrients also facilitates absorption of salt and water from the duodenum and jejunum. In SCC, patients fasted for a minimum of $6 \mathrm{~h}$ before beginning bolus saline lavage. Without fasting, the enhanced absorption of salt and water would lead to over-hydration and a lesser amount of saline solution would reach the colon, resulting in ineffective prep. In general, for all day before PEG based bowel preps,
Table 6 Patient acceptability

\begin{tabular}{llll}
\hline & SCC & HCP & $p$ value \\
\hline Solution palatability & $48 / 65(73.9 \%)$ & $38 / 68(55.9 \%)$ & 0.002 \\
Willingness to repeat & $54 / 65(83.1 \%)$ & $40 / 68(58.8 \%)$ & 0.03 \\
\hline
\end{tabular}

there is no period of fasting and patients are allowed to drink high calorie liquids until they start drinking the prep solution. We think that fasting in conjunction with dietary restriction could improve the outcome of PEG based preps also.

Anecdotally, patients reported that posture changes helped them to drink the saline solution faster. This could be explained by posture related change in gastric configuration and resultant faster emptying. The mild intensity exercises have been reported to facilitate colon prep [46].

An ideal prep requires the shortest possible dietary restriction time. In most currently available preps, patients are required to stay on clear liquids for the whole day before colonoscopy. But in SCC, patients are allowed to eat breakfast menu meal until noon.

For the best colon prep results, especially in the right colon, the interval between the prep and procedure should be short [47, 48]. Hence the bowel preparation process needs to be started depending on the scheduled time of colonoscopy [49]. We recommend starting SCC 4-6 h prior to the scheduled appointment.

Nausea was the most commonly reported adverse event in both groups. The incidence of all adverse events was similar in both groups, except for vomiting which was more common in HCP. This can be explained by PEG induced increase in gastric emptying time [50]. The lower incidence of vomiting in SCC could be due to fasting, morning only timing of the bowel prep, and faster gastric emptying in this process. Although not statistically significant, hunger cramps were also less frequently reported in the SCC.

Table 5 Electrolyte and weight changes

\begin{tabular}{|c|c|c|c|c|c|c|}
\hline & \multicolumn{3}{|c|}{$\mathrm{SCC}$} & \multicolumn{3}{|c|}{$\mathrm{HCP}$} \\
\hline & $N$ & Mean difference (SD) & $p$ value & $N$ & Mean difference (SD) & $p$ value \\
\hline Sodium & 62 & $2.55(2.85)$ & $<0.0001$ & 58 & $1.00(2.87)$ & 0.01 \\
\hline Potassium & 61 & $-0.01(0.42)$ & 0.83 & 57 & $0.03(0.55)$ & 0.70 \\
\hline Chloride & 62 & $5.16(3.00)$ & $<0.0001$ & 58 & $0.86(3.53)$ & 0.07 \\
\hline Bicarbonate & 59 & $-6.88(3.12)$ & $<0.0001$ & 54 & $-5.26(4.18)$ & $<0.0001$ \\
\hline BUN & 61 & $-3.49(3.38)$ & $<0.0001$ & 58 & $-1.67(3.19)$ & 0.0002 \\
\hline Creatinine & 61 & $0.00(0.10)$ & 0.97 & 58 & $0.07(0.14)$ & 0.0011 \\
\hline Glucose & 61 & $-11.57(18.82)$ & $<0.0001$ & 58 & $-15.53(20.44)$ & $<0.0001$ \\
\hline Weight & 63 & $-0.95(3.14)$ & 0.02 & 66 & $-2.05(3.11)$ & $<0.0001$ \\
\hline
\end{tabular}


High-volume (9-13 L) balanced electrolyte solutions (BES)/Normal saline have been successfully used for gut lavage $(25-30 \mathrm{~mL}$ per minute infusion via nasogastric tube for 4-6 h) without causing significant electrolyte imbalance or cardiopulmonary side effects in healthy individuals [51-54]. These solutions may cause deleterious fluid and electrolyte shifts in the elderly and in patients with compromised heart, liver and kidney function. The landmark study by Davis et al. [55], reported that PEG is associated with minimal water and ionic exchange, hence it will be appropriate for patients who cannot readily excrete salt and water load.

In the present study, electrolyte abnormalities of no clinical significance were reported in both groups. In SCC, normal saline was used in low volume (average $2.7 \mathrm{~L}$ ) with bolus intake. The patients drank $240-480 \mathrm{~mL}$ in $60-120 \mathrm{~s}$, almost at a ten times faster rate than the previous studies with BES/normal saline [51-54].

We presume mild hypernatremia, hyperchloremia, low bicarbonate and low BUN values within $6 \mathrm{~h}$ of bowel preparation in SCC, representing the absorbed salt and water before homeostatic mechanisms came into effect. There is little cause for concern about weight gain, fluid absorption or electrolyte imbalance in healthy patients using the SCC, especially as the solution exits the body so quickly.

The patient acceptance has been reported low for PEG based preps due to poor palatability and our study corroborates the same. A healthy person with intact homeostatic mechanisms may skip the unpleasant experience with PEG and use SCC. BPT is a potential barrier and constitutes major cost to colonoscopy procedure. One-third of working patients who undergo midweek colonoscopies miss work on additional days in anticipation of the bowel preparation [56]. SCC is an ultra-short duration process for bowel prep with least disturbance to a person's daily routine. In a cost effectiveness analysis, it can be translated into less loss of productivity. Although previous studies have reported bowel preparation efficacies in the range of $75-80 \%$, more recent studies [42-44, 57], including our own, have achieved successes of $>90 \%$. A younger patient population with no comorbidities may have contributed to our higher success rate.

\section{Limitations}

One limitation of our prep method was that nearly $70 \%$ of subjects were deemed ineligible due to-unwillingness to do Yoga, difficulty with movement ( $\mathrm{m} / \mathrm{s}$ disorders, arthritis, age $>70$ ) or exposure to saline (hypertension). Given the nearly 14 million colonoscopies performed in the US each year, even if only $30 \%$ of subjects could utilize our prep, the potential advantage of requiring a shorter bowel prep time would be clinically significant. Furthermore, patient acceptance may be $>30 \%$ in different demographics.

\section{Conclusion}

This study demonstrates that colonoscopy prep can be achieved using bolus lukewarm saline combined with sequential posture change. Due to the shorter BPT (average 1.9 h) with Shudh colon cleanse (SCC), both prep and procedure can be done on the same day.

Acknowledgments We thank our research team: Jiten Patel and Rosemary Carter RN.

Conflict of interest Guarantor of the article: Vijaypal Arya, MD president Vikalp Inc.

Open Access This article is distributed under the terms of the Creative Commons Attribution Noncommercial License which permits any noncommercial use, distribution, and reproduction in any medium, provided the original author(s) and the source are credited.

\section{References}

1. Cummings LC. Colorectal cancer screening: update 2011. Semin Oncol.. 2011;38:483-489.

2. Winawer SJ, Zauber AG, Ho MN, et al. Prevention of colorectal cancer by colonoscopic polypectomy. The National Polyp Study Workgroup. N Engl J Med. 1993;329:1977-1981.

3. National Center for Health Statistics. National Health Interview Survey, 2005. Hyattsville, Maryland: Public Health Service. Available at fttp://cdc.gov/pub/Health_Statistics/NCHS/Dataset Documentation/NHIS/2005/cancerxx_freq.pdf; 2005 Accessed September 28, 2006.

4. Holden DJ. Enhancing the use and quality of colorectal cancer screening. Evid Rep Technol Assess. (Full Rep) 2010 Feb; (190):1-195.

5. Rex DK, Johnson DA, Anderson JC, Schoenfeld PS, Burke CA, Inadomi JM. American College of Gastroenterology guidelines for colorectal cancer screening 2009 [corrected]. Am J Gastroenterol. 2009;104:739-750.

6. Strul H, Arber N. Screening techniques for prevention and early detection of colorectal cancer in the average-risk population. Gastrointest Cancer Res. 2007;1:98-106.

7. Harewood GC, Sharma VK, de Garmo P. Impact of colonoscopy preparation quality on detection of suspected colonic neoplasia. Gastrointest Endosc. 2003;58:76-79.

8. Lebwohl B, Kastrinos F, Glick M, Rosenbaum AJ, Wang T, Neugut AI. The impact of suboptimal bowel preparation on adenoma miss rates and the factors associated with early repeat colonoscopy. Gastrointest Endosc. 2011 June;73(6):1207-1214.

9. Kastenberg D, Barish C, Burack H, et al. Tolerability and patient acceptance of sodium phosphate tablets compared with 4-L PEG solution in colon cleansing: combined results of 2 identically designed, randomized, controlled, parallel group, multicenter phase 3 trials. J Clin Gastroenterol. 2007;41:54-61.

10. Parente F, Marino B, Crosta C. Bowel preparation before colonoscopy in the era of mass screening for colo-rectal cancer: a practical approach. Dig Liver Dis. 2009;41:87-95. 
11. Belsey J, Epstein O, Heresbach D. Systematic review: oral bowel preparation for colonoscopy. Aliment Pharmacol Ther. 2007;25:373-384.

12. Barnes PM, Powell-Griner E, McFann K, et al. Complementary and alternative medicine use among adults: United States, 2002. Adv Data. 2004;343:1-19.

13. Barnes PM, Bloom B. Complementary and alternative medicine use among adults: United States, 2007. National Health Statistics (CDC). Natl Health Stat Rep. 2008;12:1-23.

14. Lin M, Nahin R, Gershwin E, Longhurst J, et al. State of complementary and alternative medicine in cardiovascular, lung, and blood research: executive summary of a workshop. Circulation. 2001;103:2038-2041.

15. Galantino ML, Bzdewka TM, Eissler-Russo JL, et al. The impact of modified Hatha Yoga on chronic low back pain: a pilot study. Altern Ther. 2004;10:56-59.

16. Walton K, Schneider R, Nidich S. Review of controlled research on the transcendental meditation program and cardiovascular disease. Risk factors, morbidity, and mortality. Cardiol Rev. 2004;12:262-266.

17. Yogendra J, Yogendra HJ, Ambardekar S, et al. Beneficial effects of yoga lifestyle on reversibility of ischaemic heart disease: caring heart project of International Board of Yoga. J Assoc Physicians India. 2004;52:283-289.

18. Jacobs TL, Epel ES, Lin J, et al. Intensive meditation training, immune cell telomerase activity, and psychological mediators. Psychoneuroendocrinology. 2011;36:664-681.

19. Grant JA, Courtemanche J, Duerden EG, Duncan GH, Rainville P. Cortical thickness and pain sensitivity in zen meditators. Emotion. 2010;10:43-53.

20. Arya V, Gupta KA, Arya S. Efficacy of bolus lukewarm saline and yoga postures as a colonoscopy preparation: a pilot study. J Altern Complement Med. 2010;16:1269-1277.

21. Metabolic Equivalent (MET) values for activities in American time use survey (ATUS). National Cancer Institute, Accessed 09/15/2010. http://riskfactor.cancer.gov/tools/atus-met/met.php.

22. McHugh PR, Moran TH. Calories and gastric emptying: a regulatory capacity with implications for feeding. Am Physiol Soc. 1979;236:R254-R260.

23. Hunt JN, Macdonald I. The influence of volume on gastric emptying. J Physiol. 1954;126:459-474.

24. Hunt JN, Stubbs DF. The volume and energy content of meals as determinants of gastric emptying. J Physiol. 1975;245:209-225.

25. Erskine L, Hunt JN. The gastric emptying of small volumes given in quick succession. $J$ Physiol. 1981;313:335-341.

26. Tansy MF, Kendall FM. Experimental and clinical aspects of gastrocolic reflexes. Am J Dig Dis. 1973;18:521-531.

27. Mayer EA, Raybould HE. Role of visceral afferent mechanisms in functional bowel disorders. Gastroenterology. 1990;99: 1688-1704.

28. Jerath R, Edry JW, Barnes VA, Jerath V. Physiology of long pranayamic breathing: neural respiratory elements may provide a mechanism that explains how slow deep breathing shifts the autonomic nervous system. Med Hypotheses. 2006;67:566-571.

29. Sun WM, Houghton LA, Read NW, et al. Effect of meal temperature on gastric emptying of liquids in man. Gut. 1988;29:302-305.

30. Bateman DN. Effects of meal temperature and volume on the emptying of liquid from the human stomach. J Physiol. 1982;331: 461-467.

31. Dapoigny M, Sarna SK. Effects of physical exercise on colonic motor activity. Gastrointest Liver Physiol. 1991;260:G646-G652.

32. Burn-Murdoch R, Fisher MA, et al. Does lying on the right side increase the rate of gastric emptying? J Physiol. 1980;302:395-398.

33. Anvari M, Horowitz M, Fraser R, et al. Effects of posture on gastric emptying of nonnutrient liquids and antropyloroduodenal motility. Am Physiol Soc Am J Physiol. 1995;268:G868-G871.
34. Amidon GL, DeBrincat GA, Najib N. Effects of gravity on gastric emptying, intestinal transit, and drug absorption. $J$ Clin Pharmacol. 1991;31:968-973.

35. Gaylord SA, Palsson OS, Garland EL, et al. Mindfulness training reduces the severity of irritable bowel syndrome in women: results of a randomized controlled trial. Am J Gastroenterol. 2011;106:1678-1688.

36. Harvey RF, Read AE. Saline purgatives act by releasing cholecystokinin. Lancet. 1973;2:185-187.

37. American Society of Anesthesiologists Task Force on Sedation and Analgesia by Non-Anesthesiologists: Gross JB. Practice guidelines for sedation and analgesia by non-anesthesiologists. Anesthesiology. 2002 Apr;96(4):1004-1017.

38. Lichtenstein DR, Jagannath S, Baron TH, et al. Sedation and anesthesia in GI endoscopy. Gastrointest Endosc. 2008;68:815-826.

39. Jonas DE, Russell LB, Sandler RS, Chou J, Pignone M. Patient time requirements for screening colonoscopy. Am J Gastroenterol. 2007;102:2401-2410.

40. Jonas DE, Russell LB, Sandler RS, Chou J, Pignone M. Value of patient time invested in the colonoscopy screening process: time requirements for colonoscopy study. Med Decis Making. 2008; 2856-65.

41. DiPalma JA, Wolff BG, Meagher A, et al. Comparison of reduced volume versus four liters sulfate-free electrolyte lavage solutions for colonoscopy colon cleansing. Am J Gastroenterol. 2003;98: 2187-2191.

42. Matro R, Shnitser A, Spodik M, et al. Efficacy of morning-only compared with split-dose polyethylene glycol electrolyte solution for afternoon colonoscopy: a randomized controlled single-blind study. Am J Gastroenterol. 2010;105:1954-1961.

43. Ell C, Fischbach W, Bronisch HJ, et al. Randomized trial of lowvolume PEG solution versus standard PEG + electrolytes for bowel cleansing before colonoscopy. Am J Gastroenterol. 2008; 103:883-893.

44. Rex DK, Di Palma JA, Rodriguez R, McGowan J, Cleveland M. A randomized clinical study comparing reduced-volume oral sulfate solution with standard 4-liter sulfate-free electrolyte lavage solution as preparation for colonoscopy. Gastrointest Endosc. 2010;72:328-336.

45. Spiller RC, Trotman IF, Higgins BE, et al. The ileal brakeinhibition of jejunal motility after ileal fat perfusion in man. Gut. 1984;25:365-374.

46. Kim HS, Park DH, Kim JW, et al. Effectiveness of walking exercise as a bowel preparation for colonoscopy: a randomized controlled trial. Am J Gastroenterol. 2005;100:1964-1969.

47. Siddiqui AA, Yang K, Spechler SJ, et al. Duration of the interval between the completion of bowel preparation and the start of colonoscopy predicts bowel-preparation quality. Gastrointest Endosc. 2009;69:700-706.

48. Parra-Blanco A, Nicolas-Perez D, Gimeno-Garcia A, et al. The timing of bowel preparation before colonoscopy determines the quality of cleansing, and is a significant factor contributing to the detection of flat lesions: a randomized study. World $J$ Gastroenterol. 2006;12:6161-6166.

49. Gurudu SR, Ratuapli S, Heigh R, DiBaise J, Leighton J, Crowell M. Quality of bowel cleansing for afternoon colonoscopy is influenced by time of administration. Am J Gastroenterol. 2010; 105:2318-2322.

50. Coremans G, Vos R, Margaritis V, Ghoos Y, Janssens J. Small doses of the unabsorbable substance polyethylene glycol 3350 accelerate oro-caecal transit, but slow gastric emptying in healthy patients. Dig Liver Dis. 2005;37:97-101.

51. Hewitt J, Reeve J, Rigby $\mathbf{J}$ et al. Whole-gut irrigation in preparation for large-bowl surgery. Lancet. 1973 Aug 18;2(7825):337-340.

52. Levy AG, Benson JW, Hewlett EL, et al. Saline lavage: a rapid, effective, and acceptable method for cleansing the gastrointestinal tract. Gastroenterology. 1976;70:157-161. 
53. Postuma R. Whole bowel irrigation in pediatric patients. J Pediatr Surg. 1982;17:350-352.

54. Chattopadhyay A, Prakash B, Vepakomma D, et al. A prospective comparison of two regimes of bowel preparation for pediatric colorectal procedure: normal saline with added potassium vs. polyethylene glycol. Pediatric Surg Int. 2004;20:127-129.

55. Davis GR, Santa Ana CA, Morawski SG, Fordtran JS. Development of a lavage solution associated with minimal water and electrolyte absorption or secretion. Gastroenterology. 1980;78: 991-995.
56. Dong MH, Kalmaz D, Savides TJ. Missed work related to midweek screening colonoscopy. Dig Dis Sci. 2011;56:2114-2119.

57. Di Palma JA, Rodriguez R, McGowan J, Cleveland MV. A randomized clinical study evaluating the safety and efficacy of a new, reduced-volume, oral sulfate colon-cleansing preparation for colonoscopy. Am J Gastroenterol. 2009;104:2275-2284. 